\title{
Dalbavancin in the treatment of complicated skin and soft-tissue infections: a review
}

\author{
Jason W Bennett' \\ James S Lewis II \\ Michael W Ellis' \\ 'Department of Medicine-Infectious \\ Disease Service, Brooke Army Medical \\ Center, Fort Sam Houston, TX, USA; \\ ${ }^{2}$ Department of Medicine-Division \\ of Infectious Diseases, University of \\ Texas Health Science Center, San \\ Antonio, TX, USA
}

\begin{abstract}
Increasing rates of antimicrobial resistance among strains of Streptococcus, Staphylococcus, and Enterococcus spp. have been widely documented. At least 50\% of nosocomial Staphylococcus aureus infections in intensive care units in the US and UK are due methicillin-resistant $S$. aureus (MRSA). Drug resistance is not confined to hospitals, and community-acquired MRSA (CA-MRSA) strains are now common causes of complicated skin and soft-tissue infections (cSSTIs) in many regions. Dalbavancin is a novel parenterally administered semisynthetic lipoglycopeptide similar to the naturally produced glycopeptides vancomycin and teicoplanin. Dalbavancin features a multifaceted mechanism of action that inhibits bacterial cell wall formation by two different mechanisms that enhances its activity against a wide range of gram-positive bacteria including staphylococci, streptococci, enterococci, and some anaerobes. Additionally, dalbavancin possesses unique pharmacokinetic properties, the most significant of which is a long terminal half-life that allows for once weekly dosing. This attribute may prove to yield clinical and cost benefit. Overall, clinical trials indicate that dalbavancin is a safe, welltolerated, and effective antimicrobial agent. In the largest investigation evaluating dalbavancin for the treatment of cSSTIs, it appeared to be as effective as linezolid. Dalbavancin, which is expected to receive FDA approval in 2008, appears to be a promising new antimicrobial agent for the treatment of cSSTIs.
\end{abstract}

\section{Introduction}

The increased incidence of serious infections caused by resistant gram-positive pathogens has encouraged the development of new antimicrobial agents. Complicated skin and soft-tissue infections (cSSTIs) are broadly defined as infections of the deep soft-tissues or that require surgical intervention beyond simple incision and drainage of purulent fluid collections. Gram-positive pathogens, in particular Staphylococcus aureus and the $\beta$-hemolytic streptococci, are the most frequently implicated organisms in cSSTIs; however, in the appropriate clinical setting, other organisms including enterococci may contribute to pathogenesis (Doern et al 1999; CDC 2003; Lipsky et al 2004). Increasing rates of antimicrobial resistance among strains of Streptococcus, Staphylococcus, and Enterococcus spp. have been widely documented. The prevalence of methicillin-resistant $S$. aureus (MRSA) may vary between communities or regions; however, currently $50 \%$ of $S$. aureus isolates causing nosocomial infections in U.S. intensive care units are MRSA and similar rates for nosocomial $S$. aureus bacteremia are reported in the U.K. (Voss et al 1994; Styers et al 2006). MRSA has expanded beyond its historical niche of hospitalacquired infections in patients with traditional risk factors and has now become a major pathogen within the community (Fridkin et al 2005; Zetola et al 2005; King et al 2006; Moran et al 2006). Additionally, within certain geographic centers, community-associated MRSA (CA-MRSA) strains appear to be transitioning into nosocomial pathogens (Seybold et al 2006; Maree et al 2007). 
In the late 1980s, vancomycin-resistant enterococci (VRE) were the first pathogens identified with glycopeptide resistance (Moellering 1998). Staphylococci with reduced susceptibility to vancomycin, termed vancomycinintermediate $S$. aureus (VISA), were first reported in Japan and closely thereafter in the US (Hiramatsu et al 1997; Sieradzki et al 1999). Not long after, the first report of vancomycin-resistant $S$. aureus (VRSA) was described in a patient from Michigan; since that time, several other strains of VRSA have emerged (Sievert et al 2002; Chang et al 2003a). Besides VRSA and VISA, S. aureus strains with heteroresistance to vancomycin (hVISA) have also been described. These organisms are problematic due to subpopulations with reduced susceptibility to vancomycin in vitro that appear to increase the risk of clinical failure (Charles et al 2004). While the full clinical significance of hVISA remains unclear and both VISA as well as VRSA remain rare occurrences, these developments and trends are clinically troublesome (Deresinksi 2007).

In the face of increasing resistance in S. aureus, traditional empiric therapy with penicillinase-resistant penicillins and vancomycin has become less reliable (Deresinksi 2007). Additionally, increased scrutiny of vancomycin tissue penetration and dosing methods has fostered debate over its role as the cornerstone for serious cSSTIs infections caused by resistant gram-positive organisms (Lipsky et al 2004; Stevens et al 2005; Skhirtladze et al 2006; Mohr and Murray 2007; Deresinski 2007). Vancomycin has long been the workhorse antimicrobial therapy for resistant gram-positive infections; however, increasing resistance in cSSTI pathogens and increasing reports of clinical failures have necessitated the search for alternative agents.

Dalbavancin is a novel parenterally administered semisynthetic lipoglycopeptide developed to combat infections caused by resistant gram-positive pathogens. Dalbavancin exhibits potent in vitro bactericidal activity against gram-positive pathogens including MRSA, VISA, and non-VanA strains of VRE; and clinical data indicate that dalbavancin is a safe, effective, and well-tolerated therapy for cSSTIs (Seltzer et al 2003; Streit et al 2004; Jauregui et al 2005). US Food and Drug Administration approval is expected in 2007. This review of the English medical literature covers the pharmacologic and microbiological properties of dalbavancin with an emphasis on the available clinical data for the treatment of cSSTI.

\section{Pharmacology}

Dalbavancin (formerly BI397) is a novel semisynthetic lipoglycopeptide, and is a member of the glycopeptide antimicrobials which include: vancomycin and teicoplanin, and the investigational agents oritavancin and televancin. Due to its poor oral bioavailability, it is available only in an intravenous formulation (Van Bambeke et al 2004). Dalbavancin is derived from MDL 62,476 (formerly A-40926) which is a naturally occurring teicoplanin-like product of the actinomycete Nonomuraea spp ATCC 39727 (Beltrametti et al 2003; Technichova et al 2004). The mechanism of action of dalbavancin against gram-positive organisms is similar to other glycopeptides. Dalbavancin disrupts bacterial cell wall synthesis by binding to the C-terminal D-alanyl-D-alanine of growing peptidoglycan chains and consequently prevents transpeptidation (Finch and Eliopoulus 2005). Unlike naturally occurring glycopeptides, however, dalbavancin has the ability to dimerize and anchor into the bacterial membrane which improves stabilization and increases its affinity for the target peptidoglycan (Malabarba and Ciabatti 2001; Streit et al 2004). Investigators hypothesize that these multiple mechanisms may contribute to dalbavancin's rapid bactericidal activity against $S$. aureus (Finch et al 2005).

\section{Pharmacokinetics}

Dalbavancin possesses unique pharmacokinetic properties that distinguish it from other antimicrobial agents in the same class. The pharmacokinetics of dalbavancin are best described using a three-compartment model ( $\alpha$ and $\beta$ distributional phases followed by a terminal elimination phase). The distributional half-life ( $\mathrm{t} 1 / 2 \beta)$ that constitutes most of the clinically relevant concentration-time profile ranged from 5 to 7 days and is consistent with once-weekly dosing (Pfizer Inc. 2007). Initial in vivo studies using rat and rabbit models found extended interval dosing effective, suggesting that human trials could potentially utilize weekly dosing schedules (Cavaleri et al 2005). Data from a Phase I investigation provided further support of dalbavancin's once-weekly dosing regimen (Leighton et al 2004). In this trial, 52 healthy subjects were administered single parenteral doses of dalbavancin ranging from $140 \mathrm{mg}$ to $1120 \mathrm{mg}$, and plasma drug concentrations were measured over time. Investigators observed that following administration there was a rapid decline in the plasma drug concentration in the first 24-48 hours, representing an initial distribution phase. This was followed by a longer elimination phase with a terminal half-life measuring from 123 to 210 hours (average of 181 hours). Serum bactericidal activity was preserved against several MRSA strains tested, as plasma levels were noted to be greater than $20 \mu \mathrm{g} / \mathrm{mL}$ for 8 days in these subjects 
following a $1000 \mathrm{mg}$ dose. Additionally, in patients receiving a single dose of dalbavancin, the $C_{\max }$ increased proportionately with the administered dose; and linear regression analysis of $C_{\max }$ and AUC versus the given dose demonstrated linear dose-proportional pharmacokinetics. Taken together, this investigation supplied data supporting a once-weekly dosing regimen (Leighton et al 2004).

Dalbavancin is highly protein bound (93\%), primarily to albumin, which may account in part for its prolonged halflife (Dowell et al 2005; Pope and Roecker 2006). Despite a small free fraction in serum, dalbavancin appears to demonstrate bactericidal activity against $S$. aureus with MICs of 0.06 to $1.0 \mu \mathrm{g} / \mathrm{mL}$ (Bowker et al 2006). Dalbavancin demonstrates excellent tissue distribution and penetration in quantitative animal tissue distribution studies and in human trials (Leighton et al 2004; Cavaleri et al 2005; Nicolau et al 2007). Maximum drug concentration is achieved in most tissues within 24 hours of administration with the highest concentrations in the kidney and liver (Cavaleri et al 2005). As demonstrated in a blister-model, dalbavancin appears to have excellent skin and soft-tissue penetration (Nicolau et al 2007). Following a single $1000 \mathrm{mg}$ dose in nine healthy volunteers, the mean concentration of dalbavancin in plasma and blister fluid at day seven was noted to be $46.5 \mu \mathrm{g} / \mathrm{mL}$ and 30.3 $\mu \mathrm{g} / \mathrm{mL}$, respectively. These levels are well above the MICs for the pathogens associated with cSSTIs (Nicolau et al 2007).

Investigators have demonstrated that elimination of dalbavancin occurs by both renal and non-renal routes (Leighton et al 2004; Cavaleri et al 2005). This is in contrast to vancomycin and teicoplanin which are eliminated primarily by the kidney. In a Phase I trial, urine excretion of unaltered drug accounted for only $33.5 \%$ of the elimination of a single dose of dalbavancin which was similar to the $34.5 \%$ noted in an animal model (Leighton et al 2004; Cavaleri et al 2005). Additionally, mild renal impairment (creatinine clearance of 50 to $80 \mathrm{ml} / \mathrm{min}$ ) does not appear to alter dalbavancin's pharmacokinetic parameters (Dowell et al 2003). Nevertheless, data pertaining to side effects in patients with more severe renal impairment (creatinine clearance less than $50 \mathrm{ml} / \mathrm{min}$ ) are lacking. Further studies are needed to assess the pharmacokinetic characteristics and side effects in these patients. In terms of hepatic clearance, dose adjustments do not appear to be required in patients with mild, moderate, or severe hepatic impairment (Dowell et al 2004; Andes and Craig 2007).

\section{Pharmacodynamics}

The pharmacodynamic properties of dalbavancin have been assessed in a neutropenic murine thigh and lung model
(Andes and Craig 2007). While bacterial activity of older glycopeptides is not enhanced by higher drug concentrations above the MIC, dalbavancin has exhibited dose-dependent bactericidal activity against $S$. aureus. In a murine model developed by Andes et al, groups of mice were administered five different total doses of dalbavancin over six days (30 to $480 \mathrm{mg} / \mathrm{kg}$ for $S$. aureus) with several different dosing regimens (12-72 hours). Lengthening dosing intervals of dalbavancin appeared to improve treatment response indicating that the greatest efficacy was obtained when larger doses were administered less frequently. The data obtained from this animal model supports the clinical use of higher and less frequent dosing in order to optimize treatment efficacy (Andes and Craig 2007).

\section{Spectrum of activity Gram-positive activity}

Dalbavancin appears to possess excellent activity against a wide range of gram-positive pathogens implicated in cSSTIs: staphylococci, streptococci, and enterococci, including VanB and VanC phenotypes of VRE (Candiani et al 1999; Goldstein et al 2003; Flamm et al 2004; Streit et al 2004; Jones et al 2005; Goldstein et al 2006b; Biedenbach et al 2007). MIC data for dalbavancin against these organisms demonstrates favorable and generally lower ranges than those of vancomycin and other comparators (Candiani et al 1999; Goldstein et al 2003; Flamm et al 2004; Streit et al 2004; Jones et al 2005; Goldstein et al 2006b; Biedenbach et al 2007). Dalbavancin is also active against other grampositive pathogens including Bacillus spp., Corynebacterium spp., and Listeria spp. (Jones et al 2001; Goldstein et al 2003; Streit et al 2004; Golstein et al 2006). Additionally, in some investigations, dalbavancin appears to be more rapidly bactericidal against gram-positive organisms than vancomycin or teicoplanin (Candiani et al 1999; Jones et al 2001; Streit et al 2004).

Although no susceptibility breakpoints have been established, dalbavancin appears to demonstrate excellent activity against staphylococci. MICs for MSSA, MRSA, and Coagulase negative Staphylococcus (CoNS) range from 0.06 to 0.5 $\mu \mathrm{g} / \mathrm{mL}$ in most reports (Candiani et al 1999; Woodford 2003; Flamm et al 2004; Streit et al 2004; Jones et al 2005; Lin et al 2005; Goldstein et al 2006b). Dalbavancin displays generally lower MICs against staphylococci when compared to other agents (Candiani et al 1999; Malabarba and Ciabatti 2001; Woodford 2003; Streit et al 2004; Lin et al 2005). Dalbavancin has been tested against several isolates with reduced susceptibility to vancomycin and has generally been shown 
to display decreased activity against these strains. Three VISA isolates tested against dalbavancin were noted to have MICs ranging from 1.0 to $4.0 \mu \mathrm{g} / \mathrm{mL}$, and one VRSA isolate displayed a MIC of $<0.5 \mu \mathrm{g} / \mathrm{mL}$ (Bozdogan et al 2003; Lefort et al 2004; Goldstein et al 2007). Despite evidence suggesting less potency against VISA, the levels of dalbavancin obtained with a single $1000 \mathrm{mg}$ dose should be adequate to achieve bactericidal activity (Leighton et al 2004). Importantly, dalbavancin retains activity against $S$. aureus strains with resistance to linezolid or quinupristin/dalfopristin (Jones et al 2003; Flamm et al 2004; Streit et al 2004; Streit et al 2005). Investigators assessing resistance development in staphylococci by both direct selection and serial passage have noted no stable mutants with decreased susceptibility to dalbavancin (Goldstein et al 2007). Overall, the bactericidal activity of dalbavancin at achievable concentrations in humans throughout the proposed dosing interval suggests a low potential for the selection of resistance in patients; however, this limited data may not apply to the susceptibilities of colonizing strains exposed to prolonged post-therapy drug levels (Goldstein et al 2007).

Dalbavancin is highly active against all streptococci including S.pyogenes resistant to erythromycin and penicillin (Candiani et al 1999; Jones et al 2001; Streit et al 2004; Flamm et al 2004; Streit et al 2005; Goldstein et al 2006a). Reported MICs of $0.015-0.06 \mu \mathrm{g} / \mathrm{mL}$ are also generally lower than those of vancomycin and other comparator agents (Candiani et al 1999; Jones et al 2001; Streit et al 2004; Flamm et al 2004; Streit et al 2005; Goldstein et al 2006a). Dalbavancin is active against vancomycin-susceptible enterococci and non-VanA VRE strains. Vancomycin-susceptible enterococci have demonstrated MICs comparable to S. aureus $(0.06-0.5 \mu \mathrm{g} / \mathrm{mL})$; however, dalbavancin appears to be slightly less active against Enterococcus faecium (MIC of $0.12 \mu \mathrm{g} / \mathrm{mL}$ ) than Enterococcus faecalis (MIC of $0.06 \mu \mathrm{g} / \mathrm{mL}$ ) (Streit et al 2004). While the MICs for VanB and VanC VRE phenotypes range from 0.12 to $1 \mu \mathrm{g} / \mathrm{mL}$, the MICs for dalbavancin against VanA enterococci are considerably higher, ranging from 32 to greater than $128 \mu \mathrm{g} / \mathrm{mL}$ (Candiani et al 1999; Jones et al 2001; Jones et al 2003; Streit et al 2005). For enterococci with resistance to linezolid or quinopristin-dalfopristin, dalbavancin appears to display varrying activity depending on the individual strain (Jones et al 2003; Streit et al 2005). Taken together, dalbavancin appears to have excellent activity across the gram-positive spectrum of pathogens causing cSSTIs; however, one should not consider it as a practical therapy for gram-positive pathogens possessing the VanA gene.

\section{Anaerobic and gram-negative activity}

Dalbavancin demonstrates activity against most grampositive anaerobic pathogens although it remains inactive against most gram-negative anaerobic rods (Goldstein et al 2003; Goldstein et al 2006b). When compared to vancomycin, dalbavancin was found overall to be one to three dilutions more active in vitro against gram-positive anaerobes (Goldstein et al 2003). Similarly, in a study evaluating the effectiveness of dalbavancin against pre-treatment clinical isolates from diabetic foot infections, it was noted to be active against 120 anaerobic isolates with MICs ranging from less than $0.125 \mu \mathrm{g} / \mathrm{mL}$ to $0.5 \mu \mathrm{g} / \mathrm{ml}$ (Goldstein et al 2006b). Dalbavancin has no clinically relevant activity against gramnegative bacteria (Jones et al 2001). Dalbavancin's activity against gram-positive anaerobes may impart significant clinical benefit in the management of polymicrobial cSSTIs (Goldstein et al 2006b).

\section{Animal studies}

Dalbavancin has been studied extensively in animal models. These animal studies have demonstrated the effectiveness of dalbavancin in treating infections caused by a variety of gram-positive organisms and have helped define appropriate dosing regimens (Candiani et al 1999; Jabes et al 2004). Candiani et al employed both mouse and rat models in a multifaceted study to evaluate the use dalbavancin in septicemia, lobar pneumonia, and endocarditis (Candiani et al 1999). In the septicemia arm of their investigation, MSSA and $S$. pneumoniae were used to infect immunocompetent mice, while $S$. epidermidis and E. faecalis were used in a group of immunocompromised mice. Outcomes were measured in terms of $50 \%$ protective efficacy $\left(\mathrm{ED}_{50}\right)$ against septicemia. Dalbavancin appeared to be slightly more active than vancomycin in the immunocompetent mice. In the immunocompromised mice infected with $S$. epidermidis, dalbavancin was by far the most active agent with a significantly lower $\mathrm{ED}_{50}$; however, dalbavancin was slightly less active than teicoplanin against $E$. faecalis in the immunocompromised mice (Candiani et al 1999).

In the lobar pneumonia arm of this investigation, immunocompetent and neutropenic rats were infected with penicillin-susceptible and penicillin-resistant strains of S. pneumoniae (Candiani et al 1999). This pneumonia model demonstrated that animals that received dalbavancin had decreased bacterial load and better survival than those receiving 3 days of penicillin- $G$ regardless of penicillin susceptibility. Additionally, immunocompetent animals infected with a penicillin-resistant strain and treated with $10 \mathrm{mg} / \mathrm{kg}$ dalbavancin 
appeared to be the most efficacious regimen when compared to controls $(\mathrm{p}<0.05)$ (Candiani et al 1999).

The final arm of this investigation was a rat endocarditis model using MRSA and S. epidermidis (Candiani et al 1999). Animals were infected via a catheter placed in the left ventricle and were given either daily dalbavancin or twice daily vancomycin or teicoplanin for a total of five days. At the end of the study period and animal sacrifice, colony counts were reported in terms of CFU/g of heart tissue. Daily dalbavancin (10 $\mathrm{mg} / \mathrm{kg} /$ day) was the only antimicrobial regimen that resulted in significantly more sterile samples when compared to untreated animals $(\mathrm{p}<0.05)$. In the $S$. epidermidis arm, drug regimens were similar in both outcomes and were both significantly better than untreated controls $(\mathrm{p}<0.05)$. Overall, the results from this group of elaborate animal experiments demonstrated that dalbavancin was at least as effective as vancomycin or teicoplanin and provided results prompting further investigation (Candiani et al 1999).

LeFort et al also evaluated dalbavancin with a rabbit endocarditis model using $S$. aureus strains susceptible to vancomycin and teicoplanin (MICs of 2 and $4 \mu \mathrm{g} / \mathrm{mL}$, respectively) and strains with reduced susceptibility to both glycopeptides (MICs of 8 and $16 \mu \mathrm{g} / \mathrm{mL}$, respectively) (LeFort et al 2004). In this model, rabbits were infected with S. aureus and then treated with dalbavancin $10 \mathrm{mg} / \mathrm{kg}$ once daily for 4 days or with a single $40 \mathrm{mg} / \mathrm{kg}$ dose of the agent. Vegetation homogenates were plated and counted as CFU/g of vegetation. Both dalbavancin treatment groups obtained a significant reduction in the colony count after therapy when compared to the untreated control group ( $p<0.01)$. Additionally, the once-daily dalbavancin dose was more effective than the single dose regimen. This endocarditis model provided evidence that dalbavancin has comparable activity to vancomycin against $S$. aureus with reduced glycopeptide susceptibility (LeFort et al 2004).

In another rabbit model, investigators compared the ability of dalbavancin and vancomycin to prevent $S$. aureus colonization of subcutaneously implanted intravenous catheters (Darouiche and Mansouri 2005). Investigators harvested these subcutaneously inserted catheters seven days after they had been implanted and inoculated with $S$. aureus. In this study, vancomycin performed no better than a saline control infusion in preventing catheter colonization. In contrast, dalbavancin prevented colonization in $28 \%$ of the catheters compared to $47 \%$ in the vancomycin-treated rabbits $(p=0.07)$. Although these results were not significant, this study suggested that there may be a future role for dalbavancin in the prevention and treatment of device-related infection (Darouiche and Mansouri 2005).

In a rodent granuloma pouch model, investigators compared the activities of dalbavancin, linezolid, and vancomycin against experimental MSSA and MRSA bacterial infections (Jabes et al 2004). In this study, suspensions of $S$. aureus were inoculated into rat granuloma pouches and antibiotic regimens were started shortly after infection. Aliquots of pouch exudates were removed, diluted, and plated in order to assess colony growth in terms of $\mathrm{CFU} / \mathrm{ml}$ of exudate. In this set of experiments, single dose dalbavancin $(2.5,5$, and $10 \mathrm{mg} / \mathrm{kg}$ doses $)$ was compared to multiple doses of vancomycin or linezolid. Data from this study showed that dalbavancin was effective at reducing the bacterial load of MSSA at all doses when compared to the control group. Additionally, a dose-dependent reduction in MRSA bacterial load occurred with dalbavancin at the $5 \mathrm{mg} / \mathrm{kg}$ and $10 \mathrm{mg} / \mathrm{kg}$ single doses. The single $10 \mathrm{mg} / \mathrm{kg}$ dalbavancin $/ \mathrm{kg}$ dose reduced the viable MRSA count in pouch exudates by more than $2 \log \mathrm{CFU} / \mathrm{mL}$, and regrowth was prevented for up to 120 hours. To achieve comparable results with vancomycin, four $100-\mathrm{mg} / \mathrm{kg}$ intramuscular doses were needed. Data from this model demonstrated not only the efficacy of single-dose dalbavancin, but also that it could be administered less frequently than both linezolid and vancomycin (Jabes et al 2004).

\section{Clinical efficacy}

The safety and efficacy of dalbavancin has been examined in three clinical trials (Seltzer et al 2003; Raad et al 2005; Jauregui et al 2005). These trials include: one Phase II investigation that examined tolerability and efficacy in catheterrelated gram-positive bloodstream infections (CR-BSI) (Raad et al 2005); and two trials (one Phase II and one Phase III) that evaluated dalbavancin in the treatment of skin and soft-tissue infection (SSTI) (Seltzer et al 2003; Jauregui et al 2005).

\section{Phase II trials}

Seltzer et al first conducted an open label, randomized, controlled, Phase II multi-center trial examining one-dose and two-dose dalbavancin versus standard-of-care therapy for the treatment SSTI (Seltzer et al 2003). Standard-ofcare therapy was chosen prior to randomization by the investigators and included vancomycin, cephalosporins, clindamycin, piperacillin-tazobactam, or linezolid. If anaerobic or additional gram-negative antimicrobial coverage was thought necessary by investigators, patients could receive aztreonam, ceftazidime, or metronidazole. Clinical response was measured at day 10 for one-dose dalbavancin, at day 20 
for two-dose dalbavancin, and on the last day of treatment for the comparator regimens. Additionally, patients were again assessed fourteen days following the completion of either therapy. Patients were excluded from the entry into the investigation for the following reasons: impaired renal function (creatinine clearance less than $50 \mathrm{~mL} / \mathrm{min}$ ), recent treatment for SSTI (within 7 days of study entry), self-limited infections, compromised vascularity, documented osteomyelitis, and history of vancomycin hypersensitivity (Seltzer et al 2003).

A total of 62 patients were enrolled in the study and received at least 1 dose of a study medication (Seltzer et al 2003). The three treatment groups included: those receiving a single $1100 \mathrm{mg}$ intravenous dose dalbavancin (20 patients); those receiving a $1000 \mathrm{mg}$ dose of dalbavancin followed by a $500 \mathrm{mg}$ dose seven days later (21 patients); and those receiving the comparator therapy which was administered for seven to twenty-one days (21 patients). Comparator regimens (standard-of-care of care) were determined by the investigators prior to randomization and included vancomycin, cephalosporins (ceftriaxone, cefazolin, cephalexin), clindamycin, piperacillin-tazobactam, and linezolid. Eleven patients were excluded from the clinically evaluable population for the following: requiring antimicrobial therapy for a non-SSTI or use of corticosteroids ( 5 patients); no evidence of a grampositive infection at baseline (3 patients); non-compliance with follow-up (2 patients), and SSTI with compromised vascular supply (1 patient). Approximately two-thirds of the patients enrolled in the investigation had undergone surgical intervention prior to randomization. The three study groups had similar success rates at the end of therapy and at the 2-week follow-up visit. There was a trend toward a more favorable response in the two-dose dalbavancin group. In intention to treat analysis at the end of therapy, success rates were $91 \%$ (19 of 21 ) for two-dose dalbavancin, 75\% (15 of 20) for one-dose dalbavancin, and $81 \%$ (17 of 21) for the comparator group. Sixty-one organisms were cultured from 42 patients in the microbiological intent to treat population with the majority (47 of 61 isolates) being $S$. aureus. Success rates for patients with MRSA infection were $80 \%$ (4 of 5) for two-dose dalbavancin, $50 \%$ (3 of 6 ) for one-dose dalbavancin and $50 \%$ (1 of 2 ) for the comparator group. A similar rate of success was noted in patients from whom streptococcal species were isolated. Failures in any of the groups were not explained by antibiotic resistance or lack of in vitro activity of the antimicrobial agent against the bacterial isolate (Seltzer et al 2003).

Investigators noted drug-related adverse events in 55\% of patients in the one-dose dalbavancin group, $48 \%$ of patients in the two-dose dalbavancin group, and $57 \%$ in the comparator regimen group (Seltzer et al 2003). Of note, no patients receiving dalbavancin exited the study due to drug-related adverse events. Due to the investigation's small sample size, no statistical analysis was reported; nevertheless, this study did provide human clinical evidence suggesting that two-dose dalbavancin could be effective in the treatment of SSTI (Seltzer et al 2003).

In another Phase II clinical trial, Raad et al evaluated the safety and efficacy of dalbavancin for the treatment of CRBSI caused by gram-positive pathogens (Raad et al 2005). In this randomized, controlled, open-label multi-center trial, patients with presumed CR-BSI were assigned treatment with either two doses of dalbavancin (a $1000 \mathrm{mg}$ dose on day one, followed by $500 \mathrm{mg}$ on day 8 ) or vancomycin (1000 mg twice daily) for 14 days. Catheter removal was required for all cases of confirmed $S$. aureus infection and recommended but not required for CoNS infection. Patients were excluded from the study if they had significant renal or hepatic impairment, had received immunosuppressive therapy, had experienced prolonged neutropenia, had prior treatment with an antibiotic active against gram-positive organisms within 48 hours of initiation of the study medication, or had a pulmonary artery catheter. Investigators also excluded patients who had had $S$. aureus bacteremia during the previous three months thought to be from a non-catheter-related source (eg, endocarditis, osteomyelitis, infected prosthetic material, deep abscess, or septic thrombophlebitis). At the investigators' discretion, a gram-negative active agent (aztreonam or ceftazidime) and an anaerobic active agent (metronidazole) were permitted based on clinical suspicion. The primary outcome measured was test-of-cure which was assessed by combining clinical and microbiologic response at a follow-up visit 18-24 days after completing therapy (Raad et al 2005).

Seventy-five patients were enrolled in the investigation, 67 of whom were included in the intent to treat population (Raad et al 2005). With regard to CR-BSI etiology, 54 bacterial isolates were recovered from 51 study patients. Of these 54 isolates, CoNS (26 isolates) was the most frequently isolated pathogen. $S$. aureus was the next most common pathogen (23 isolates), 14 of which were MRSA. Five E. faecalis isolates were also recovered. Overall, bacterial isolates did not appear to be significantly different at baseline in the microbiologically confirmed intent to treat population; however, more patients in the vancomycin group, $32.1 \%$ ( 9 of 28 patients) had MRSA infection compared to $19.2 \%$ ( 5 of 26 patients) in the dalbavancin group. In the microbiologic intent to treat analysis, overall success at the 
follow up visit was $87 \%$ (20 of 23 ) (95\% CI: $73.2 \%-100 \%$ ) for the dalbavancin group and 50\% (14 of 28) (95\% CI: $31.5 \%-68.5 \%$ ) for the vancomycin group. Microbiologic success at the follow-up visit as defined by eradication or presumed eradication of the organism was $95.7 \%$ ( 22 of 23 patients) for those receiving dalbavancin and $78.6 \%$ (22 of 28 patients) for those receiving vancomycin (Raad et al 2003). A follow-up study subsequently characterized the blood isolates obtained from this trial (Goldstein et al 2006a). The MIC results were less than $0.25 \mu \mathrm{g} / \mathrm{mL}$ for all of the isolates obtained from both groups, which included CoNS, S. aureus, and E. faecalis (Goldstein et al 2006a).

While these results appear to suggest significant benefit of dalbavancin over therapy with vancomycin, there are several important limitations to this study. First, this study was designed only to assess safety and efficacy of dalbavancin, not to determine superiority. Second, vancomycin (except in the case of significant allergy) would be considered a secondline agent for the treatment of MSSA bacteremia as it has been shown to be inferior to antistaphylococcal penicillins or first-generation cephalosporins for the treatment of MSSA bacteremia (Chang et al 2003b; Stryjewski et al 2007). Third, interpretation of the data is made difficult by the disparity in the number of MRSA-infected patients in each group (Raad et al 2003). Lastly, although the investigators used a standard dosing regimen, vancomycin serum drug levels were not reported; higher vancomycin levels may have altered outcome (Mohr and Murray 2007).

\section{Phase III trials}

In the only published Phase III investigation, Jauregui et al compared dalbavancin with linezolid for the treatment of cSSTIs (Jauregui et al 2005). In this double-blind, randomized, multicenter trial, 854 adult patients were enrolled with a 2:1 randomization scheme. The 571 patients in the dalbavancin group received a $1000 \mathrm{mg}$ dose on day one of therapy and a second dose of $500 \mathrm{mg}$ on day 8 . The 283 patients in the linezolid group received $600 \mathrm{mg}$ twice daily for 14 days, with at least 24 hours of initial intravenous therapy. Investigators defined cSSTI as an infection that involved deeper soft-tissue, required significant surgical intervention (eg, major abscess, major burns, surgical wound infection, and extensive or ulcerating cellulitis), or as a SSTI known or thought to be caused by MRSA. Additionally, patients were required to demonstrate at least two local signs and/or symptoms of cSSTI. Patients were excluded from participation if they had osteomyelitis, septic arthritis, were anticipated to require more than two surgical interventions during the study, or would require additional antimicrobial therapy for concomitant infections. Empiric use of aztreonam and/or metronidazole was permitted at the investigators' discretion if a polymicrobial infection was suspected. The primary outcome measured was clinical success in all evaluable patients at a test-of-cure visit 14 days ( \pm 2 days) after completing study medication. For study purposes, each dose of dalbavancin represented seven days of therapy (Jauregui et al 2005).

At baseline, there were no significant demographic differences between the two groups and they appeared to be well matched in terms of types of infection and underlying medical history (Jauregui et al 2005). At least one grampositive pathogen was recovered at baseline in $64 \%$ of patients. Ninety percent of the cultured isolates from each group were $S$. aureus ( $51 \%$ in each group were MRSA) and all remaining isolates were streptococcal species (Groups A, C, and G streptococcus, and Viridans streptococcus spp). Among patients who were clinically evaluable at the test-of-cure visit, the dalbavancin and linezolid groups achieved clinical success in $88.9 \%$ and $91.2 \%$, respectively. Additionally, MRSA eradication rates at the test-of-cure visit were similar in both groups, with 91\% cured in the dalbavancin group and $89 \%$ cured in the linezolid group. This investigation demonstrated that dalbavancin was well tolerated and as effective (noninferior) to linezolid in the treatment of cSSTIs. Additionally, dalbavancin exhibited excellent efficacy against MRSA, suggesting that it could be an alternative to vancomycin or linezolid for patients in whom these agents would be contraindicated (Jauregui et al 2005).

\section{Adverse events}

Dalbavancin appears to be well tolerated in animal studies and in all clinical investigations. The clinical trials evaluating dalbavancin have not demonstrated any statistically significant differences in adverse events in patients receiving dalbavancin versus comparator agents (Seltzer et al 2003; Raad et al 2005; Jauregui et al 2005). Indeed, there have been very few adverse reactions severe enough to result in termination of dalbavancin in any clinical trial to date. Neither ototoxity, nephrotoxicity, nor Red man syndrome has been described with dalbavancin therapy (Seltzer et al 2003; Jauregui et al 2005; Raad et al 2005).

In a Phase I dose escalation study in healthy volunteers Leighton et al demonstrated no serious adverse events associated with dalbavancin administration (Leighton et al 2004). Additionally, in doses up to $1120 \mathrm{mg}$, no dose effect in terms of adverse events or laboratory abnormalities was noted. In this investigation, at least one treatment-associated adverse 
event was reported by $67 \%$ of the 39 study participants who received dalbavancin. In general, these adverse events were mild and did not result in study drug discontinuation. The most common events were subjective pyrexia $(50 \%)$, headache $(25 \%)$, and nausea $(6 \%)$. Oral temperature did not exceed $37.5^{\circ} \mathrm{C}$ in any participant at any point during the study, and subjects in the placebo group reported similar reactions with regard to pyrexia (38\%) and headache (31\%). One subject developed an asymptomatic, transient, mild transaminitis (less than 5 times the upper limit of normal) following administration of $350 \mathrm{mg}$ dalbavancin which was not noted in any subject receiving higher doses; and one subject in both the dalbavancin and placebo groups experienced a transient mild hyperglycemia (Leighton et al 2004). Finally, no audiological changes or vestibular toxicity have been noted in any subjects receiving dalbavancin (Campbell et al 2003; Leighton et al 2004).

In a Phase II trial, Seltzer et al noted similar tolerability (Seltzer et al 2003). In this investigation dalbavancin-related adverse events were reported by 11 patients (55\%) receiving single dose dalbavancin, 10 patients (48\%) receiving 2 doses of dalbavancin, and 12 patients (57\%) receiving the comparator regimens. They found no clinically concerning laboratory abnormalities as a result of treatment in any treatment group. No patients discontinued dalbavancin nor left the study due to an adverse event (Seltzer et al 2003).

In a separate Phase II trial conducted by Raad et al where 33 patients were administered $1000 \mathrm{mg}$ of dalbavancin followed by a $500 \mathrm{mg}$ dose one week later, the most common treatment-related adverse events included: diarrhea (21\%), hypotension (21\%), constipation (18\%), pyrexia (18\%), and oral candidiasis (12\%) (Raad et al 2005). Adverse events appeared to be more frequent in the dalbavancin group but were mild and did not lead to discontinuation of dalbavancin therapy or withdrawal from the study (Raad et al 2005).

Similar to other investigations, in a Phase III clinical trial comparing dalbavancin to linezolid for the treatment of cSSTIs, Jauregui et al reported mild side effects and good tolerability with dalbavancin therapy (Jauregui et al 2005). The most common dalbavancin-associated adverse events in this study were primarily gastrointestinal: nausea $(3.2 \%)$, diarrhea $(2.5 \%)$, and vomiting (1.9\%). One serious adverse event was reported in the dalbavancin group, but this was considered not to be treatment related. This adverse event was a transient and mild leukopenia. Adverse events related to treatment were overall more frequent in the linezolid group $(32.2 \%)$ than the dalbavancin group (25.4\%) (Jauregui et al 2005). This trial along with the other preclinical and clinical trials has demonstrated that dalbavancin is well tolerated without significant toxicity. It is important to remember that clinical studies have excluded patients who report a history of vancomycin or other glycopeptide allergy. Further clinical studies are needed to determine long term safety and drug effects.

\section{Drug interactions}

There has been a paucity of published data on drug-drug interactions with dalbavancin. An in vitro study evaluated combinations of dalbavancin with representatives of nine other classes of antimicrobial agents (oxacillin, gentamicin, clindamycin, levofloxacin, rifampicin, vancomycin, quinupristin/dalfopristin, linezolid, and daptomycin) for antagonism and synergy against staphylococci, enterococci and streptococci, including resistant isolates (Johnson et al 2006). The investigators detected no antagonism between dalbavancin and any of the other agents. Synergy was observed when dalbavancin was combined with oxacillin and tested against four strains of $S$. aureus; however, no synergy was observed with gentamicin (Johnson et al 2006). These in vitro observations warrant further investigation.

It is clear that non-renal mechanisms are important in the clearance of dalbavancin; however, there is no evidence suggesting that the cytochrome P450 enzyme system is involved in its metabolism (Leighton et al 2004; Buckwalter and Dowell 2005). A review of 532 patients who had received dalbavancin during several clinical trials noted that $79 \%$ had received concomitant medications that possessed a potential for significant interaction through either inhibition or induction of the cytochrome P450 enzyme system (Buckwalter and Dowell 2005). These medications included: acetaminophen, aztreonam, fentanyl, metronidazole, furosemide, proton pump inhibitors, midazolam, and simvastatin. These classes of medications did not appear to clinically impact the clearance of dalbavancin (Buckwalter and Dowell 2005).

\section{Conclusion}

The emergence of resistant pathogens responsible for cSSTIs in addition to increased clinical failures with vancomycin has driven the development of new antimicrobial agents. Dalbavancin is a novel parenterally administered semisynthetic lipoglycopeptide that possesses excellent activity against a wide range of gram-positive pathogens that cause cSSTIs. This agent's unique pharmacokinetic profile, primarily attributed to its long half-life, allows for once-weekly dosing. Phase II and Phase III trials have demonstrated that dalbavancin is a safe, effective, and well-tolerated antimicrobial for the treatment of cSSTI. Its clinical effectiveness is enhanced 
by its once-weekly dosing. Once-weekly dosing may prove to be cost effective and clinically beneficial in certain patient populations. The FDA is expected to approve dalbavancin in 2008. At a time when new antimicrobials are needed, dalbavancin appears to be a promising new antimicrobial agent for the treatment of cSSTIs.

\section{Disclaimer}

The opinions or assertions contained herein are the private views of the authors and are not to be construed as official or reflecting the views of the Department of the Army, the Department of Defense or US Government. The authors are employees of the US government. This work was prepared as part of their official duties and, as such, there is no copyright to be transferred.

\section{References}

Andes D, Craig WA. 2007. In vivo pharmacodynamic activity of the glycopeptide dalbavancin. Antimicrob Agents Chemother, 51:1633-42.

Beltrametti F, Lazzarini A, Brunati C, et al. 2003. Production and characterizatio of monochlorinated and dechlorinated A40926 derivatives. $J$ Antibiot, 56:773-82.

Biedenbach DJ, Ross JE, Fritsche TR, et al. 2007. Activity of dalbavancin tested against Staphylococcus spp and $\beta$-hemolytic Streptococcus spp isolated from 52 geographically diverse medical centers in the United States. J Clin Microbiol, 45:998-1004.

Bowker KE, Noel AR, MacGowan AP. 2006. Pharmacodynamics of dalbavancin studied in an in vitro pharmacokinetic system. $J$ Antimicrob Chemother, 58:802-5.

Bozdogan B, Esel D, Whitener C, et al. 2003. Antibacterial susceptibility of a vancomycin-resistant Staphylococcus aureus strain isolated at the Hershey Medical Center. J Antimicrob Chemother, 52:864-8.

Buckwalter M, Dowell JA. 2005. Population pharmacokinetic analysis of dalbavancin, a novel lipoglycopeptide. J Clin Pharmacol, 45:1279-87.

Campbell KC, Kelly E, Targovnik N, et al. 2003. Audiologic monitoring for potential ototoxicity in a phase I clinical trial of a new glycopeptide antibiotic. J Am Acad Audiol,14:157-68.

Candiani G, Abbondi M, Borgonovi M, et al. 1999. In-vitro and in-vivo antibacterial activity of BI 397, a new semi-synthetic glycopeptide antibiotic. J Antimicrob Chemother, 44:179-92.

Cavaleri M, Riva S, Valagussa A, et al. 2005. Pharmacokinetics and excretion of dalbavancin in the rat. J Antimicrob Chemother, 55(Suppl 2):ii31-5.

Centers for Disease Control and Prevention National Nosocomial Infections Surveillance (NNIS) System. 2003. NNIS system report, data summary from January 1992 through June 2003, issued August 2003. Am J Infect Control, 31:481-98.

Chang FY, Peacock JE Jr, Musher DM, et al. 2003b. Staphylococcus aureus bacteremia: recurrence and the impact of antibiotic treatment in a prospective multicenter study. Medicine, 82:333-9.

Chang, S, Siever DM, Hageman JC, et al. 2003a. Infection with Vancomycinresistant Staphylococcus aureus containing the vanA resistance gene. N Engl J Med, 348:1342-7.

Charles PG, Ward PB, Johnson PD, et al. 2004. Clinical features associated with bacteremia due to heterogenous vancomycin-intermediate Staphylococcus aureus. Clin Infect Dis, 38:448-51.

Darouiche RO, Mansouri MD. 2005. Dalbavancin compared with vancomycin for prevention of Staphylococcus aureus colonization of devices in vivo. $J$ Infect, 50:206-9.

Deresinski S. 2007. Counterpoint: Vancomycin and Staphylococcus aureusan antibiotic enters obsolescence. Clin Infect Dis, 44:1543-8.
Doern GV, Jones RN, Pfaller MA, et al. 1999. Bacterial pathogens isolated from patients with skin and soft-tissue infections: frequency of occurrence and antimicrobial susceptibility patterns from the SENTRY Antimicrobial Surveillance Program (United States and Canada, 1997). SENTRY Study Group (North America). Diagn Microbiol Infect Dis, 34:65-72.

Dowell JA, M. Buckwalter, E. Seltzer, et al. 2005. Dalbavancin penetration into skin supports once weekly dosing. (Abstract 895). 15th European Congress of Clinical Microbiology and Infectious Diseases (ECCMID). Copenhagen, Denmark 2005, April 2-5.

Dowell JA, Seltzer E, Buckwalter M, et al. 2004. The Pharmacokinetics of Dalbavancin (DAL) in Subjects with Mild, Moderate or Severe Hepatic Impairment (HI). Abstracts of the 44th Interscience Conference of Antimicrobial Agents and Chemotherapy. Washington, DC, 2004. 2.

Dowell JA, Seltzer E, Stogniew M, et al. 2003. Dalvavancin dosage adjustment not rcquired for patients with mild renal impariment (abstract P1224). Clin Microbiol Infect, 9(Suppl 1):291.

Finch RG, Eliopoulos GM. 2005. Safety and efficacy of glycopeptide antibiotics. J Antimicrob Chemother, 55(Suppl 2):ii5-13.

Flamm RK, Draghi DC, Karlowsky JA, et al. 2004. Activity of dalbavancin against clinical isolates of staphylococci and streptococci from the U.S. and Europe. Abstracts of the Forty-fourth Interscience Conference on Antimicrobial Agents and Chemotherapy. Washington, DC, USA, 2004. 173

Fridkin SK, Hageman JC, Morrison M, et al. 2005. Methicillin-resistant Staphylococcus aureus diseases in three communities. N Engl J Med, 352:1436-44.

Goldstein BP, Jones RN, Fritsche TR, et al. 2006a. Microbiologic characterization of isolates from a dalbavancin clinical trial for catheter-related bloodstream infections. Diagn Microbiol Infect Dis, 54:83-7.

Goldstein EJ, Citron DM, Merriam CV, et al. 2003. In vitro activities of dalbavancin and nine comparator agents against anaerobic gram-positive species and corynebacteria. Antimicrob Agents Chemother, 47:1968-71.

Goldstein EJ, Citron DM, Warren YA, et al. 2006b. In vitro activities of dalbavancin and 12 other agents against 329 aerobic and anaerobic gram-positive isolates recovered from diabetic foot infections. Antimicrob Agents Chemother, 50:2875-9.

Goldstein BP, Draghi DC, Sheehan DJ, et al. 2007. Bactericidal activity and resistance development profiling of dalbavancin. Antimicrob Agents Chemother, 51:1150-4.

Hiramatsu K, Aritaka N, Hanaki H, et al. 1997. Dissemination in Japanese hospitals of strains of Staphylococcus aureus heterogeneously resistant to vancomycin. Lancet, 350:1670-3.

Jabes D, Candiani G, Romano G, et al. 2004. Efficacy of dalbavancin against methicillin-resistant Staphylococcus aureus in the rat granuloma pouch infection model. Antimicrob Agents Chemother, 48:1118-23.

Jauregui LE, Babazadeh S, Seltzer E, et al. 2005. Randomized, double-blind comparison of once-weekly dalbavancin versus twice-daily linezolid therapy for the treatment of complicated skin and skin structure infections. Clin Infect Dis, 41:1407-15.

Johnson DM, Fritsche TR, Sader HS, et al. 2006. Evaluation of dalbavancin in combination with nine antimicrobial agents to detect enhanced or antagonistic interactions. Int $J$ Antimicrob Agents, 27:557-60.

Jones RN, Biedenbach DJ, Johnson DM, et al. 2001. In vitro evaluation of BI 397, a novel glycopeptide antimicrobial agent. $J$ Chemother, 13:244-54.

Jones RN, Fritsche TR, Sader HS, et al. 2005. Antimicrobial spectrum and potency of dalbavancin tested against clinical isolates from Europe and North America (2003): initial results from an international surveillance protocol. J Chemother, 17:593-600.

Jones RN, Sader HS, Fritsche TR. 2003. Dalbavancin (DAL; formerly BI397) activity against selected populations of antimicrobial-resistant gram-positive pathogens. (Abstract 172). Abstract of the 41st Annual Meeting of the Infectious Diseases Society of America. San Diego: IDSA, 2003.

King MD, Humphrey BJ, Wang YF, et al. 2006. Emergence of communityacquired methicillin-resistant Staphylococcus aureus USA 300 clone as the predominant cause of skin and soft-tissue infections. Ann Intern Med, 144:309-17. 
Lefort A, Pavie J, Garry L, et al. 2004. Activities of dalbavancin in vitro and in a rabbit model of experimental endocarditis due to Staphylococcus aureus with or without reduced susceptibility to vancomycin and teichoplanin. Antimicrob Agents Chemother, 48:1061-4.

Leighton A, Gottlieb AB, Dorr MB, et al. 2004. Tolerability, pharmacokinetics, and serum bactericidal activity of intravenous dalbavancin in healthy volunteers. Antimicrob Agents Chemother, 48:940-5.

Lin G, Credito K, Ednie LM, et al. 2005. Antistaphylococcal activity of dalbavancin, an experimental glycopeptide. Antimicrob Agents Chemother, 49:770-2.

Lipsky BA, Berendt AR, Deery HG, et al. 2004. Diagnosis and treatment of diabetic foot infections. Clin Infect Dis, 39:885-910.

Malabarba A, Ciabatti R. 2001. Glycopeptide derivatives. Curr Med Chem, 8:1759-73.

Maree CL, Daum RS, Boyle-Vavra S, et al. 2007. Community-associated methicillin-resistant Staphylococcus aureus isolates causing healthcareassociated infections. Emerg Infect Dis,13:236-42.

Moellering RC. 1998. Vancomycin-resistant Enterococci. Clin Infect Dis, 26:1196-9.

Mohr JF and Murray BE. 2007. Point: Vancomycin is not obsolete for treatment of infection caused by methicillin-resistant Staphylococcus aureus. Clin Infect Dis, 4:1536-42.

Moran GJ, Krishnadasn A, Gorwitz RJ, et al. 2006. Methicillin-resistant $S$. aureus infections among patients in the emergency department. N Engl J Med, 355:666-74.

Nicolau DP, Sun HK, Seltzer E, et al. 2007. Pharmacokinetics of dalbavancin in plasma and skin blister fluid. J Antimicrob Chemother, epublished Jul 12, 2007.

Pfizer Inc. 2007. Dalbavancin [prescribing information]. New York, NY: Pfizer Inc.

Pope SD, Roecker AM. 2006. Dalbavancin: a novel lipoglycopeptide antibacterial. Pharmacotherapy, 26:908-18.

Raad I, Darouiche R, Vazquez J, et al. 2005. Efficacy and safety of weekly dalbavancin therapy for catheter-related bloodstream infection caused by gram-positive pathogens. Clin Infect Dis, 40:374-80.

Seltzer E, Dorr MB, Goldstein BP, et al. 2003. Once-weekly dalbavancin versus standard-of-care antimicrobial regimens for treatment of skin and soft-tissue infections. Clin Infect Dis, 37:1298-303.

Seybold U, Kourbatova EK, Johnson JG, et al. 2006. Emergence of community-associated methicillin-resistant Staphylococcus aureus USA300 genotype as a major cause of health care-associated blood stream infections. Clin Infect Dis, 42:647-656.
Sieradzki K, Roberts RB, Haber SW, et al. 1999. The development of vancomycin resistance in a patient with methicillin-resistant Staphylococcus aureus infection. $N$ Engl J Med, 340:517-23.

Sievert DM, Boulton ML, Stolzman G, et al. 2002. Staphylococcus aureus resistant to vancomycin-United States, 2002. MMWR Morb Mortal Wkly Rep, 51:565-7.

Skhirtladze K, Hutschala D, Fleck T, et al. 2006. Impaired target site penetration of vancomycin in diabetic patients following cardiac surgery. Antimicrob Agents Chemother, 50:1372-5.

Stevens DL, Bisno AL, Chambers HF, et al. 2005. Practice guidelines for the diagnosis and management of skin and soft-tissue infections. Clin Infect Dis, 41:1373-1406.

Streit JM, Fritsche TR, Sader HS, et al. 2004. Worldwide assessment of dalbavancin activity and spectrum against over 6,000 clinical isolates. Diagn Microbiol Infect Dis, 48):137-43.

Streit JM, Sader HS, Fritsche TR, et al. 2005. Dalbavancin activity against selected populations of antimicrobial-resistant Gram-positive pathogens. Diagn Microbiol Infect Dis, 53:307-10

Stryjewski ME, Szczech LA, Bejnajmin DK Jr, et al. 2007. Use of vancomycin or first-generation cephalosporins for the treatment of hemodialysis dependent patient with methicillin-susceptible Staphylococcus aureus bacteremia. Clin Infect Dis, 44:190-6.

Styers D, Sheehan DJ, Hogan P, et al. 2005. Laboratory-based surveillance of current antimicrobial patterns and trends among Staphylococcus aureus: 2005 status in the United States. Ann Clin Microbiol Antimicrob, 5:2.

Technikova-Dobrova Z, Damiano F, Tredici SM, et al. 2004. Design of mineral medium for growth of Actinmadura sp. ATCC 39727, produce of the glycopeptide A40926: effects of calcium ions and nitrogen sources. Appl Microbil Biotechnol, 65:671-7.

Van Bambeke F, Van Laethem Y, Courvalin P, et al. 2004. Glycopeptide antibiotics: from conventional molecules to new derivatives. Drugs, 64:913-36.

Voss A, Milatovic D, Wallrauch-Schwarz C, et al. 1994. Methicillinresistant Staphylococcus aureus in Europe. Eur J Clin Microbiol Infect Dis, 13:50-55.

Woodford, N. 2003. Novel agents for the treatment of resistant gram-positive infections. Expert Opin Investig Drugs, 12:117-37.

Zetola N, Francis JF, Nuermberger EL, et al. 2005. Community-acquired methicillin-resistant Staphylococcus aureus: an emerging threat. Lancet Infect Dis, 5:275-86. 